\title{
Hepatic Hemorrhage, CTCAE
}

National Cancer Institute

\section{Source}

National Cancer Institute. Hepatic Hemorrhage, CT CAE. NCI Thesaurus. Code C143540.

A disorder characterized by bleeding from the liver. 